\title{
Observations sur quelques formes religieuses de loyalisme, particulières à la Gaule et à la Germanie romaine.
}

\section{Pir J. Toutain.}

Il est aujourdliui reconnu que le culte rendu par les populations de l'empire romain à la divinité de Rome, à celle d'Anguste ou des empereurs soit vivants soit morts, était moins une vraie religion qu'une forme religieuse de loyalisme politique. Par la s'exprimaient l'attachement des provinciaux ¿̇ la puissance romaine et leur fidélité anx souverains en qui se personnifiait cette puissance. Ce culte nous est connu sous trois formes principales. Il se célébrait dans chaque province, al nom de toutes les cités de la province; il se célébrait dans chaque cité, au nom de tous les habitants de la ville; enfin dans maintes régions du monde romain, surtont en Italie, en Espagne et en Narbonnaise, les seviri Augustales adressilient à la divinité impériale les hommages des aftranchis et des petites gens. ${ }^{1}$ ) Sous sa forme provinciale et sous sa forme municipale, ce culte était public; le prêtre provincial et le prêtre municipal de Rome, d'Auguste ou des empereurs étaient des personnages très importants, revêtus d'une dignité officielle. Quant anx Augustales, le culte qu'ils célébraient était, sinon officiel et public, du moins collectif. D'autre part cette triple organisation du culte paraît avoir été liée très étroitement à la vie municipale: en effet, les membres des assemblées provinciales étaient les délégués des diverses cités de la province; le culte municipal n'existait que dans les centres de population constitués vraiment en communes; enfin la corporation des Augustales ne pouvait se fonder et vivre que dans un milieu urbain.

Or il y eut dans l'empire romain de vastes régions où la vie municipale se développa moins qu'en Italie ou en Bétique: telles furent, par

1) Sur l'organisation de ces diverses formes du culte de Rome et d'Auguste, voir surtout: P. Gurraud, Les Assemblées provinciales dans l'empire romain, Paris, 1887; E. Beurler, Essai sur le culte rendu aux entpereurs romains, Paris, 1890; F. Mourdot, Essai sur l'histoire de l'Augustalité dans l'empire romain, Paris, 1895. 
exemple, presque toute la Gaule chevelue et les denx Germanies. Ce n'est pas à dire qu'il n'y en̂t pas de villes dans ces contrées; mais, antant que nous pouvons en juger par les documents épigraphiques, ces villes furent bien moins qu'ailleurs les organes essentiels de la vie publique; les institutions municipales n'y acquirent pas l'éclat et l'importance qu'elles eurent dans d'autres provinces. Aussi n'y trouve-t-on que peu de traces du culte municipal de Rome et d'Auguste ou des empereurs, du moins sous sa forme habituelle: les inscriptions nomment très peu de flamines d'Augtrste, et ceux qu'elles mentionnent vivaient précisément dans les parties de la Gaule qui subirent le plus l'influence romaine, par exemple chez les Edluens, ${ }^{1}$ ) chez les Sénons, ${ }^{2}$ ) ḋ Noviodunum (Nyon), ${ }^{3}$ ) chez les Helvètes, ${ }^{4}$ ) chez les Vellaves, ${ }^{5}$ ) les Ségusiaves, ${ }^{6}$ ) les Bituriges Cubes, ${ }^{7}$ ) a Bordeanx. ${ }^{5}$ ) Pour tout le nord de la Gaule, un seul prêtre municipal est nommé chez les Morins $;^{9}$ ) les Germanies n'en ont jusqu'i présent fourni ancun. Les Augustales sont également très rares dans les trois Gaules et sur les bords du Rhin: s'ils existaient à Lyon ${ }^{10}$ ) et antour de Lyon, ${ }^{11}$ ) chez les Helvètes, ${ }^{12}$ ) les Eduens, ${ }^{13}$ ) les Sénons, ${ }^{14}$ ) les Bituriges Cubes, ${ }^{15}$ ) les Nédiomatriques, ${ }^{16}$ ) plus al nord et à l'est, on en rencontre à peine quelques mentions isolées à Xanten, ${ }^{17}$ ) Nimèg'te, ${ }^{1}$ ) Aix la Chapelle, ${ }^{19}$ ) Mayence, ${ }^{2 v}$ ) Worms, ${ }^{21}$ ), Rotenbourg (Würtemberg)..2)

On se tromperait néanmoins si l'on concluait de ces observations que les Gaulois et les tribus germaniques soumises a la domination romaine n'avaient pas revêtu leur loyalisme d'une forme religiense. Il est au contraire tout in fait remarquable que précisément dans ces régions, où la

1) CIL., XIII, 2585.

2) Ibid, 2940.

3) Moumsen, Inscr. Helvet., 118, 119, ef. 112.

4) Id., ibid., 179, 189, 193, 194, 142, 143.

5) CIL., XIII, 1577.

6) Ibid., 1642.

7) Ibid., 1376 et 1377 .

8) Ibid., 602.

9) Vamlant, Epigr. de la Morinie, n. 22.

10) CIL., XIII, 1735,1751 et surtout 1985 et suiv.

11) Ibid., 2543.

12) Моммsen, Inscr. Helvet., 121, 133, 149, 156, 177, 187 et suiv., 223.

13) CIL., XIII, 2584, 2652, 2669.

14) Ibid., 2926.

15) Ibid., 1194.

16) Robert et Cagnat, Epigr. gallo-romaine de la Moselle, II, p. 16.

17) Brambace, CIRh., 10.

18) Id., ibid., 82.

19) Td., ibid., 628.

20) Id., ibid., 1316.

21) Id., ibid., 904.

22) Id., ibid., 1628, 1683 . 
civilisation gréco-romaine pénétra moins profondément qu'ailleurs, des hommages très nombreux furent adressés soit à la divinité d'Auguste ou des empereurs, soit an dien qui protég'eait tout spécialement l'empire, Jupiter Optimus Maximus. Ces hommages n'avaient presque jamais de caractère public; ils émanent à peu près partont et toujours d'un sentiment de dévotion individuelle; en second lieu, les inscriptions qui nous les ont fait connaître, et les monuments figurés qui nous ont apporté sur ces vieilles religions des témoignages souvent très précieux, attestent qùe dans la pensée de ces Gaulois et de ces Germains leurs anciens cultes nationaux ou locaux vivaient en parfait accord avec ces cultes nouveaux; ì nos yeux cette union des divinités est une des formes les plus originales que prit dans l'empire romain le loyalisme des provinciaux.

Tel est le phénomène i la fois religieux et historique que nous nons proposons d'étudier ici brièvement, d'abord dans les trois Gaules, puis dans les Germanies.

\section{I.}

\section{Les trois Gaules.}

Dans les trois Gaules, mais principalement en Aquitaine et en Lugdunaise, ont été trouvés en grand nombre des textes votifs et des dédicaces, où sont mentionnés côte à côte soit Anguste soit les Augustes et une divinité nationale ou locale.

La divinité impériale est tantôt appelée tout simplement Augustus; par exemple:

Adidoni et Augusto. Se.x. Telonius Musicus d. s. p. p. ${ }^{1}$ ), tantôt Numen Augusti oul au pluriel Numina Augustorum: ainsi

Num(ini) Aug. et deo Mercuri Dumiati Matutinus Victorinus d. d. ${ }^{2}$ ) et Numinib(us) Augustor(um) deo Volkano M. Gemel. Secundus et C. Sedat. Florus actor(es) vicanor(um) Portens(ium) tribunal cum locis ex stipe contata posuerunt.:

Parmi ces inscriptions trois émanent non point de cités proprement dites, de communes organisées suivant les institutions romaines, mais de tribus gauloises on de villages. Ce sont:

$1^{\circ}$ Augusto sacrum et Genio civitatis Bit(uriges) Viv(isci).4)

$2^{\circ}$ Numinibus Aug(ustorum), fanum Plutonis Andecamulenses de suo posuer(unt). $\left.{ }^{5}\right)$

$3^{\circ}$ Numinibus Augustorum et Junonibus vicani Neriomagienses. $\left.{ }^{6}\right)$
1) CIL., XIII, 1575.
2) Ibid., 1523.
3) Ibid., 1306 (Nantes).
4) Ibid., 566 (Bordeaux).
5) Ibid., 1449 (Limoges).
6) Ibid., 1374 (Néris Ies Bains). 
A Bordeaux, la divinité impériale est associée à une divinité locale, le Genius Civitatis; it Néris les Bains, elle est jointe anx Junones, déesses apharentées aux Matres, aux Matronae, aux Suleviae, anx Fatae, et dont le nom romain ne dissimule pas le caractère gaulois. A Limoges, le cas est plus curienx: les Numina dugnstorum sont invoqués ì propos d'un fanum Plutonis, comme si la construction de ce sanctuaire était placée sous la protection des divinités impériales.

A Entrains (Nièvre), les ouvriers en bronze de cette localité unissent sur un ex-voto le nom du dieu Anguste í celui du Dous Borvo et Candidus:

Aug. sacr. Deo Borvoni et Candido acrari sub cura Leonis et Marciani ex voto; acrarii dona (verunt) $\left.{ }^{1}\right)$

Mais le plus souvent de tels hommages sont individuels; ils pronvent que, même en l'absence d'un culte municipal organisé ou d'une corporation d'Augustates, le culte de lin divinité impériale s'était répandu parmi les populations gauloises.

Le nom d'Auguste ou des Augustes se trouve associé

c) à des divinités nationales, tlont le nom est accompagné ou non d'une épithète locale:

Mercure:

[Numini] Aug. et Mercurio v. s. l. m. Tiz. Cl. Potit[us ...]."

Mercurio et Augusto sacrum.")

Numini Augustor. Mercurio sacr. ${ }^{4}$ )

Auy. sacr. Deo Mercurio Censorinus Pusellini filius ex voto. ${ }^{5}$ )

Aug. sacr. Deo Mercurio Am .... .us Celsus Ambiorigis (f.) ex voto.')

Num. Aug. signum Mercur. cum sua aede $)$....

Num. Aug. et deo Mercuri Dumiati Matutinus Victorinus d. d. ${ }^{\mathrm{s}}$ )

Mars:

Num. Aug. et Marti Mogetio Gracchus Ategnutis fil. v. s. l. $\mathrm{m}^{\text {") }}$

N. Aug. Deo Marti Segomoni Dunati Cassia Saturnina ex voto. ${ }^{10}$ )

Aug. saer. Deo Varti Cicollui et Litavi P. Altius Paterchus v. s. l. m. ${ }^{11}$ )

Aug. sacr. Marti Boluinno et Duna(ti) C. Domit. Virilis decurio ...... ${ }^{12}$ )

1) CIL., XIIT, 2901.

2) Ibid., 1124 (Poitiers).

3) Ibid., 1514 (Auvergne).

4) Ibid, 2501 (env. de Belley).

5) Ibid., 2631 (pays des Eduens).

6) Ibid., 2891 (env. de Sens).

7) Ibid., 3177 (Lisieux).

8) Ibid., 1523 (Puy de Dôme).

9) Ibid., 1193 (Bourges).

10) Xbid., 2532 (Env. de Belley).

11) Ibid, 2887 (Env, de Langres).

12) Ibid., 2899 (Sens). 
Aug. sacr. Mart(i) Boluinn(o) L. Gabinius Severus donum dedit. $\left.{ }^{1}\right)$

Aug. Marti Mulloni signum cum suo templo et ornamentis omnitnus suo et Toutillae fil. nomine Agdovirus Morici fil. $v$ s. l. m. ${ }^{2}$ )

Numinibus Augustorum Deo Marte ..... usso Tullianus v. s. l. m.3) Apollon:

Num. Au[g.] et Genio Apollinis Atepomari Jul. Atrectus Craxanti fil. et .Jul. Gnatus Atrecti fil. d. s. d. $\left.{ }^{4}\right)$

Aug. Apollini sacr. Cosmis Lucan(i) fil. $\left.{ }^{5}\right)$

Numinib. August. Deo Apollini C. Nonius Euposius ex voto muro et scandula cinxit. $\left.{ }^{6}\right)$

Aug. sacr. Deo Apollini. ${ }^{\top}$ )

Ninerve:

Aug. sacr. Dea $(e)$ Miner $\left.[v] a(e) \ldots{ }^{5}\right)$

Numinibus Augustorum et deae Minervae M. Petroni $[u s . . . ? C]$ amilli fili. aetem ....d.s.p. p. c.")

Aug[usto sacr. Decie Hiner $]$ vae et ..... murnm ..... et a[re$\left.\mathrm{cm}] \ldots . .{ }^{10}\right)$

Vulcain:

Numinib. Augustor. Deo Vollkano M. Gemel. Secundus et C. Sedat. Florus actor. vicanor. Portens. tribunal cum locis ex stipe conlata posuerunt. ${ }^{11}$ )

Aug. Deo Volkano porticum cum campo consecratam L. Mart.... M. Lucceins Genialis vicanis Portensibus conces(serunt). $\left.{ }^{12}\right) \cdot$

$[N u] m$. Aug. [deo] Volk(ano). [Ma]rcius ..... cus [Mar $]$ celli $[$ fili $]$ us [v. s.] $\left.l . m^{13}\right)$

Les déesses Mères:

Numinib. Aug. Matris Augustis C. Nonius .. ${ }^{14}$ )

Rosmerta:

Aug. sacr. Deae Rosmertae Cne. Cominius Candidus et Apronia Avitilla v. s. l. $m .{ }^{15}$ )
1) CIL, XIII, 2900 (Sens).
2) Ibid., 3101 (Nantes).
3) Ibid., 3103 (Nantes).
4) Ibid, 1318 (Bourges).
5) Ibid., 3073/4 (Tours).
6) Ibid., 1730 (Lyon).
7) Ibid., 2898 (Sens).
8) Ibid., 2892 (Sens).
9) Ibid., 3075 (Tours).
10) Ibid., 3104 (Nantes).
11) Ibid., 3106 (Nantes).
12) Ibid., 3107 (Nantes).
13) Ibid., 3164 (Vieux).
14) Ibid., 1764 (Lyon).
15) Ibid., 2831 (pays des Edueus). 
Epona:

Aug. sacr. Deae Eponae Marcellus Haturi t. incol(a) d. s. v. s. m. ${ }^{1}$ ) Augusto sacrum. Deae Eponae Counonius Icotasgi fil templum cum suis ornamentis omnibus de suo donavit $l . m .^{2}$ )

Sirona:

Num. Aug. De(ae) Strona(e) Ca. Magrusa. lib. v. s. l. m.")

$\beta)$ à des divinités locales:

Numin. Aug. et deo Telon(2)? . . . . .4)

[Numini]b Aug. et Nerio deo ... etc. . .5)

Augu(sto) sacru $(m)$. Deo Brixantu propitiu ...6)

Aug. sacr. Deo Albio et Damonae Sex. Mart. Cociliani f. ex jussu ejus s. l. $\left.m .{ }^{7}\right)$

Aug. sacr. Deae Seq(uanae) Fl. Flavialis pro sal. Fl. Iuna. nep sui ex voto v. s. l. $\mathrm{m.}^{8}$ )

Aug. sacr. Deae Clutö̈dae et Ucanis Masrvensibus Mediusacer Medianni f. murum inter arcus duos cum suis ornamentis d. s. p. $f .{ }^{9}$ )

Aug. sacr. Deue Icauni 7. Tetricius African(us) d. s.d.d. d. ${ }^{0}$ )

Aug. Acionnae sacrum. Capellus Illiomari f. porticum. "i)

Aug. Rudiobo sacrum cur. Cam. ... d. s. p. d. Ser. Esumagius Sacrovir, Ser . . . S Sever us. ${ }^{12}$ )

[A]ug. Deo Gisaco Tauricius Agricola de suo posuit. ${ }^{13}$ )

Comme on peut le voir par cette énumération, c'était une habitude chez beaucoup de Gaulois d'invoquer la divinité impériale, Auguste ou les Augustes, en même temps que leurs dieux nationaux et locaux; presque toujours même, c'était le nom d'Auguste qu'ils inscrivaient tout d'abord sur les dédicaces ou les ex-voto dont ils ornaient leurs sanctuaires. De telles manifestations religieuses n'avaient point de caractère officiel; elles exprimaient donc des sentiments spontanés. Nous y voyons la preuve certaine que la puissance impériale était acceptée de bon gré par ces provincianx. Leurs prières, leurs actions de grâces se partageaient entre l'Auguste ou les Augustes, et la divinité nationale ou locale, dont ils

1) CIL., XIII, 2903 (Entrains).

2) Ibid., 2902 (Entrains).

3) Ibid., 3143 (pays de Corseul).

4) Ibid., 948 (Périgueux).

5) Ibid., 1376/1377 (Néris les Bains).

6) Ibid., 2812 (près d'Autun).

7) Ibid., 2840 (pays des Eduens).

8) Ibid., 2862 (Sources de la Seine).

9) Ibid., 2895 (Sens).

10) Ibid., 2921 (Auxerre, près de l'Yonne).

11) Ibid., 3063 (Orléans).

12) Ibid., 3071 (Orléans).

13) Ibid., 3197 (Evreux).

Beitrige z. alten Geschichte II 2. 
étaient les fidèles. Ils unissaient ainsi, en une seule et même invocation, la religion de l'empire et les anciens cultes de leur race. Cette union nous semble être la forme même de leur loyalisme, forme religieuse et populaire, partant sincère et dénuée de toute flatterie hypocrite.

II.

\section{Les Germanies.}

Dans les deux provinces de Germanie inférieure et de Germanie supérieure, c. a. d. dans presque tont le bassin du Rhin, le culte, par lequel semble s'être le mieux exprimée la fidélité des provinciaux, fut non point le culte de la divinité impériale, mais celui du grand dieu Capitolin, Jupiter Optimus Maximus. Tandis que dans les trois Gaules, les dédicaces à Augustus, au Numen Aug., aux Numina Augg. sont très fréquentes, elles font absolument défaut sur les bords du Rhin; au contraire le nom de Jupiter Optimus Maximus, très souvent abrégé en $J$. O.M., se lit sur des inscriptions extrêmement nombreuses. Parfois les trois divinités du Capitole, Jupiter Optimus Maximus, Juno Regina, Minerva sont invoquées ensemble; souvent Juno Regina apparaît à côté de Jupiter; mais dans le plus grand nombre des cas, c'est à Jupiter seul, à Jupiter Optimus Maximus, que s'adressent les prières et les remerciements des fidèles. Or ce culte de Jupiter Optimus Maximus, soit associé aux autres divinités capitolines, soit seul, présente un caractère analogue à celui du culte d'Auguste ou des Augustes dans les trois Gaules. Dans les Germanies comme dans les provinces voisines, l'accord s'établit entre la divinité romaine, protectrice de l'empire, et les dieux du pays conquis. A la période de lutte, succéda l'entente et l'amitié.

Ce rapprochement des divinités se manifeste surtout dans deux catégories de documents qu'il convient de distinguer. Parmi les dédicaces et les inscriptions votives qui ont été trouvées dans les Germanies, et sur lesquelles se lisent à la fois le nom de Jupiter Optimus Maximus et celui d'une divinité locale ou nationale, plus ou moins nommément désignée, il en est beancoup qui émanent d'officiers, de sous-officier's et de soldats du corps d'occupation, en voici quelques exemples:

J. O. M. et Genio loci M. Aurelius Ursulus bf. cos. pro se et suis $v$. s. l. m. Frusco it(erum) et Dextro cos. (225 ap. T. C.)')

J. O. M. et Genio loci L. Septimius Bellus sig(nifer) leg. XXII Pr(imigeniae), optio Navali(orum) v. s. l. l. m. Saturnino et Gallo cos. (198 ap. J. C. $\left.)^{2}\right)$

In h(onorem) d(omus) d(ivinae) J. O. M., Genio loci et Fortunae dis deabusque Emeritius Sextus miles legionis XXII Pr(imigeniae) p. f. Severia-

1) Brambacir, OTRh, 430 (env. de Cologne).

2) Id., ibid., 1302 (Mayence). 
nae, bf. cos., pro se et suis posuit v. s. l. l. m., Maximo et Aeliano cos. idibus Januaris (223 ap. J. C. $)^{1}$ )

J. O. M. et Genio loci et Rheno Cl. Marcellinus bf. cos. v. s. l. m. Imp. Commodo VI cos. (190 ap. J. C. $\left.)^{2}\right)$

J. O M. (et) Her(culi) Sax(ano) Sex. Donnius Vindex >leg. X G(eminae) p. fid. et commititones v. s. l. m. ${ }^{3}$ )

l. . $\left.m^{4}\right)$

J. O. M. et Marti Caturigi genio loci C. Jul. Quietus bf. cos. v. s.

II est bien certain que ces soldats, sous-officiers et officiers ne pelvent pas être considérés comme de simples provinciaux; par le fait même qu'ils servaient dans les légions romaines, ils étaient en quelque manière des représentants de la puissance impériale. Mais n'est-il pas très caractéristique de les voir, eux, de leur côté, invoquer, en même temps que le dieu du Capitole, les divinités locales du pays où ils se trouvaient en garnison? N'est-ce pas lì une preuve qu'il n'y avait entre les anciennes populations et l'armée de Rome qui les avait vaincues, aucun sentiment de haine et de discorde? L'accord, l'union des divinités et des cultes ne se comprendrait pas si les habitants de la Germanie romaine ne s'étaient pas montrés sujets respectueux et fidèles du gouvernement impérial.

Aussi bien leur loyalisme se manifesta sous une forme, qui nous parait être très intéressante à étudier de près.

Dans tout le bassin moyen du Rhin, entre Bâle et Mayence, ainsi que dans les vallées đu Neckar et de la Moselle, ont été trouvées plusieurs séries de monuments archéologiques, que l'on a d'abord étudiées chacune à part, puis que l'on a rapprochées, parce que certaines découvertes récentes ont démontré que ces monuments, d'aspect si différent, n'étaient que les parties d'un senl et même ensemble. Ces séries sont: les autels dits des quatre dieux, ${ }^{5}$ ) les autels dits des sept dieux de la semaine, ${ }^{6}$ ) les colonnes appelées colonnes de Jupiter, ${ }^{i}$ ) enfin les groupes, presque intacts ou très fragmentaires, connus sous le nom de groupes du Cavalier et de l'Anguipède. ${ }^{\S}$ ) Grâce aux trouvailles faites depuis un demi-siècle

1) Braxnacu, CIRh., 1574 (Kannstadt, Wïrtemberg).

2) Id., ibid., 647 (Remagen, près de Coblenz).

3) Id., ibid., 651 (Brohl, près de Bonn); cf. 652, 657, 662, 665 et Freudenberg, Das Denkmal des Hercules Saxanus im Brohtthal, Bonn, 1862.

4) Brambach, CIRh., 1588 (Böckingen, Wurtemberg).

5) $\mathrm{H}_{\mathrm{AU}}$, Die Viergottersteine, dans la Westdentsche Zeitschrift, $\mathrm{X}$ (1891).

6) HAUG, Die Wochengöttersteine, dans la Westdeutsche Zeitschrift, IX (1890).

7) F. Hextaner, Juppitersculen, dans la Westdeutsche Zeitschrift, IV (1885); cf. Korrespondenzblatt der W. Z., 1890 no 65 et 1901 no 21 .

8) Aug. Prost, Les travaux consacrés au Groupe de l'Anguipède et du Cavalier justac'en 1891, dans les Mémoires de la Société des Antiquaires de France, 1891. 
environ à Heddernheim, ${ }^{1}$ ) à Merten, $\left.{ }^{2}\right)$ surtout à Schierstein,"3) les érudits ont pu reconstituer trois monuments dont la forme et la décoration sont des plus originales. La partie inférieure de chacun de ces monuments est constituée par un piédestal carré, dont chaque face porte en relief plus ou moins accentué une image de dieu ou de déesse; sur ce piédestal repose un socle soit circulaire, soit hexagonal ou octogonal, parfois orné de sept images qui représentent les divinités présidant aux sept jours de la semaine. Ce socle supporte une colonne, que domine un chapiteau oi se voient des motifs de décoration peu habituels dans l'art gréco-romain, tels que des têtes humaines ou des serpents; enfin le chapiteau sert luimême de base à un groupe de sculpture, qui représente un personnage à cheval foulant aux pieds un anguipède renversé par terre. Il ne saurait subsister aucun donte sur la reconstitution de l'ensemble; aussi pouronsnous conclure à l'existence de tels monuments partout oì a été retrouvé même un seul des éléments essentiels dont il se compose, autel dit des quatre dienx, colonne de Jupiter, groupe plus ou moins bien conservé du Cavalier et de l'Anguipède.

Ce n'est point ici le lieu d'entreprendre une étude analytique et approfondie de ces monuments. Nous nous bornerons a présenter certaines conclusions, qui nous paraissent peu contestables.

Ce n'est point de la Grèce ni de Rome que sont originaires ces monuments. Nulle part sur les bords de la Méditerranée il n'en a été jusqu'à présent trouvé de pareils ni même d'analogues. La région, où ont été recueillis des vestiges de ces monuments, est nettement délimitée: le centre en est la Germanie supérieure; elle s'étend d'une part jusqu'an limes Germanicus, qui séparait les Champs Décumates de la Germanie demeurée indépendante, d'autre part jusqu'en Bretagne et en Auvergne. Mais entre cette région et l'Italie, aucun inclice n'a encore été relevé qui témoigne d'une relation quelconque entre ces monuments et le foyer de la civilisation gréco-romaine. C'est ailleurs qu'il faut chercher, si l'on veut trouver la provenance de ces monuments. C'est en Germanie. Nous savons, par des documents du Moyen-Age, que plusieurs tribus germaniques, en particulier les Saxons, les Francs, les Angles, et les habitants de la Suède, avant leur conversion au christianisme, élevaient à leurs anciennes divinités des idoles en forme de colonnes. On connait par exemple l'Trminsûl des Saxons, les colonnes de Roland chez les Francs, les colonnes

1) Korrespondenzblatt der Westdeutschen Zeitschrift, IV no 3; ef. HAuG, Die Viergöttersteine, no 61-62.

2) A. Prost, Le monument de Merten, dans la Revue archéologique, 1879, 1.

3) Korrespondenzblatt der Westdeutschen Zeitschrift, VIII, n. 149. - Cf. Fronscпùтz, Die Gigantensäule von Schierstein, dans les Annalen des Vereins fü̈r Nassauische Alterthumskunde, t. XXII (1890), p. 119 et suiv. 
de Thor en Suède, les colonnes d'Athelstan en Angleterre, etc. ${ }^{1}$ ) Ces colonnes étaient sans donte plus grossières, plus simples de forme que les monuments dont nous nous occupons; mais cenx-ci nous paraissent procéder de celles-là, malgré l'influence gréco-romaine qui se trahit dans leur agencement et leur décoration.

C'est encore à la même conclusion que nous arriverons, si nous examinons quelles sont les divinités, dont les images sont sculptées sur les piédestaux. Assurément l'aspect général, le costume, les attributs de ces divinités sont empruntés ì peu près exclusivement ¿̇ la mythologie et $\dot{a}$ l'art gréco-romains: les dieux y sont Jupiter, Mars, Mercure, Minerve, Hercule, Apollon; parmi les divinités féminines, le premier rang de beancoup appartient à une déesse, qui le plus souvent est figurée sous les traits de Junon, mais qui rappelle aussi parfois Demèter-Cérès, Proserpine, Vénus ou Cybèle, déesse qui personnifie probablement la fécondité de la nature. Or il est tout à fait remarquable que dieux et déesse soient ici précisément les divinités dont César et Tacite nous disent qu'elles étaient spécialement adorées par les Gaulois et les Germains; nous pouvons en effet admettre que la déesse, nommée par Tacite tantôt Tsis, tantôt Terra Mater ou Nerthus, a été assimilée par les Germains romanisés soit à Junon, soit parfois à Cerès, à Vénus, à Cybèle. Quant à Jupiter, Mars, Mercure, Minerve, Hercule, Apollon, ils sont nommément designés par les deux historiens romains. ${ }^{2}$ )

En ce qui concerne le groupe famenx du Cavalier et de l'Anguipède, on sait combien d'interprétations diverses les érudits en ont données.") A notre avis, le problème doit être divisé. Il est très probable, comme le vent en particulier M. Heuzer, que le motif a été emprunté à l'art hellénique; mais cela ne prouve nullement que l'idée, la légende ou le symbole exprimé ait la même origine. Nous sommes, pour notre part, très disposé à voir dans ce motif la représentation grecque on gréco-romaine d'un mythe d'origine germanique. Ce mythe, c'est la lutte de Thor contre les Géants, variante du grand mythe naturiste, que contiennent toutes les religions indo-européennes, védique, iranienne, grecque, latine, celtique, etc.

Ainsi, malgré l'aspect tout classique de certains détails, malgré la physionomie hellénique ou latine des personnages divins, malgré le caractère romain de la décoration, ces monuments nons semblent appartenir à un tout autre cycle religieux que le polythéisme de la Grèce et de Rome. Ils sont en relations très étroites avec la Germanie. Ce sont, sous une forme romanisée, les équivalents des colonnes, qu'élevaient les tribus païennes des Saxons, des Angles, des Francs et des Goths de Scandinavie.

1) Grism, Deutsche Mythologie, p. 100 et suiv.

2) César, De bello Gallico, VI, 16 et suiv.; Tacite, De Germania, 9 et 40 .

3) Cf. A. Prost, Les travaux consacrés, etc. 
$204 J$. Toutain, Observations sur quelques formes roligicuses de loyalisme etc.

Or sur quelques-uns de ces monuments se lisent des dédicaces et des inscriptions votives; le dieu, anquel ces monuments étaient dédiés, n'est antre que Jupiter Optimus Maxımus, le dien du Capitole, le protecteur tout puissant de Rome et de l'empire romain. Parfois le nom de Jupiter Optimus Maximus est accompagné de celui de Juno Regina. Presque toujours les dédicants sont des individus, de petites gens, qui déclarent dans l'inscription que le monument a été élevé chez eux, dans leur propre domaine, in su. ${ }^{\mathrm{D}}$ )

Nous nous trouvons ici en présence d'un plénomène à la fois historique et religieux, dont la portée et le sens sont pour nous tout à fait comparables à la portée et au sens de l'accord qui s'était établi en Ganle entre le culte d'Anguste et les cultes garlois nationaux et locaux. Dans la Germanie romaine, on consacrait au grand dieu Capitolin des monuments inspirés par les traditions mythologiques et rituelles de la Germanie. On adressait an Jupiter romain des hommages, on hui dédiait des monuments qu'auparavant on adressait et on dédiait sans doute aux grandes divinités du panthéon germanique. Ici encore l'entente et l'union s'étaient faites. Elles ne se seraient pas faites si les Germains annexés $\dot{2}$ l'empire n'avaient pas loyalement accepté la domination de Rome. Ce loyalisme, essentiellement politique, trouva sa forme religieuse particulière dans l'attribution ì Jupiter Optimus Maximus des monuments que nous venons d'étudier.

1) Brambach, CTRh., no 1106, 1310, 1317, 1318, I322, 1328, 1705; Korrespondenzblatt der Westdeutschen Zeitschrift, 1889 no 118 et 149; Hetrner, Juppitersáulen, p. 370 n. 2, p. 372 n. 8 et 9 etc., etc. 